\title{
Electrochemotherapy in Kaposi sarcoma: A systematic review
}

\author{
MARTINA FERIOLI ${ }^{1}$, ANDREA GALUPPI $^{1}$, MILLY BUWENGE $^{1}$, SILVIA CAMMELLI $^{1}$, \\ ANNA MYRIAM PERRONE ${ }^{2}$, GABRIELLA MACCHIA ${ }^{3}$, FRANCESCO DEODATO ${ }^{3}$, \\ SAVINO CILLA $^{4}$, ALICE ZAMAGNI ${ }^{1}$, FRANCESCA DE TERLIZZI ${ }^{5}$, LUCA TAGLIAFERRI ${ }^{6}$, \\ PIERANDREA DE IACO ${ }^{2}$ and ALESSIO GIUSEPPE MORGANTI ${ }^{1}$
}

\author{
${ }^{1}$ Radiation Oncology Center, Department of Experimental, Diagnostic and Specialty Medicine-DIMES, \\ ${ }^{2}$ Oncologic Gynaecology Unit, Department Medical and Surgical Sciences (DIMEC), University of Bologna, \\ S. Orsola-Malpighi Hospital, I-40138 Bologna; ${ }^{3}$ Radiotherapy Unit and ${ }^{4}$ MedicalPhysic Unit, Gemelli Molise Hospital, \\ Fondazione Policlinico Universitario A. Gemelli, IRCCS, I-86100 Campobasso; ${ }^{5}$ Scientific and Medical Department \\ IGEA S.p.A., I-41012 Carpi (Mo); ${ }^{6}$ Fondazione Policlinico Universitario ‘A. Gemelli’ IRCCS, UOC di Radioterapia \\ Oncologica, Dipartimento di Diagnostica per Immagini, Radioterapia Oncologica ed Ematologia, I-00168 Roma, Italy
}

Received May 12, 2020; Accepted September 9, 2020

DOI: $10.3892 / \mathrm{mco} .2021 .2226$

\begin{abstract}
Kaposi sarcoma (KS) is a rare angioproliferative disorder, which is usually associated with human herpesvirus- 8 infection. Electrochemotherapy (ECT) may be an option in the treatment of KS skin lesions due to the high response rate noted in neoplastic lesions of different histological types. The aim of the present systematic review was to analyse the available evidence on using ECT in the treatment of KS skin lesions. Tumor response, local control and toxicity were analyzed. In the three included studies, the complete response rate was $65-100 \%$ and the overall response rate in all studies was $100 \%$. The treatment was well tolerated with mild and transient toxicity. However, further studies are required to fully analyze long-term disease control and to compare ECT with other local therapies used for KS.
\end{abstract}

\section{Introduction}

Kaposi sarcoma (KS) is a rare angioproliferative disorder associated with the human herpes virus-8 (HHV-8) infection. The latter is a necessary but not sufficient condition in the multifactorial pathogenesis of the disease. Four epidemiological forms have been identified as follows: Classic, endemic (in Africa), iatrogenic (in patients undergoing immuno-suppressive therapies), and epidemic (in HIV-patients) (1). KS frequently manifests itself with

Correspondence to: Dr Martina Ferioli, Radiation Oncology Center, Department of Experimental, Diagnostic and Specialty Medicine-DIMES, University of Bologna, S. Orsola-Malpighi Hospital, Via Giuseppe Massarenti 9, I-40138 Bologna, Italy E-mail:m.ferioli88@gmail.com

Key words: electrochemotherapy, Kaposi sarcoma, systematic review, skin lesions, human herpesvirus- 8 skin lesions (macules, nodules, plaques) and can lead to the development of visceral metastases. In the advanced stage of the disease, $\mathrm{KS}$ is associated with a more unfavourable prognosis $(1,2)$. Moreover, $\mathrm{KS}$ can be indolent or may have a rapidly progressive and life-threatening behaviour (3).

Standard treatment options for KS are limited. Particularly, skin lesions can be treated with several local therapies, such as surgery, radiotherapy, cryosurgery, $\mathrm{CO}_{2}$-laser, or intra-lesional chemotherapy (3). Moreover, antiretroviral drugs are used in the HIV-related form of KS, while metastatic KSs are generally treated with chemotherapy, which is based on pegylated liposomal doxorubicin and paclitaxel (3).

Electrochemotherapy (ECT) is an option used in the treatment of KS skin lesions due to its high response rate in neoplastic lesions of different histological types (4). ECT is the combination of electroporation and systemic administration of chemotherapeutic agents. According to the ESOPE guidelines (4), the only absolute contraindications to ECT are pregnancy, lactation, and allergy or hypersensitivity to the used drugs. Electroporation can produce transient pores in the cell membrane allowing the entry of hydrophilic molecules into the cytoplasm (5). The two drugs that have been successfully tested for this purpose are bleomycin and cisplatin. Their cytotoxicity has increased to 1,000 and 80 times by electroporation, respectively (6). Furthermore, preclinical and in vivo studies have shown a similar efficacy of ECT in neoplastic lesions from different primary tumors (7). Moreover, if necessary, patients can also undergo repeated ECT treatments, as long as the lifetime bleomycin dose does not exceed 400,000 IU, without precluding any subsequent local or systemic treatments.

ECT was initially introduced for the treatment of primary and metastatic skin tumors (4). However, ECT has been suggested to be particularly effective in treating KS skin lesions. In addition to the chemosensitizing effect, electroporation produces a 'vascular lock' in the treated volume that is potentially able to control an angio-proliferative neoplasm, such as KS (8). 
However, the existing evidence on the efficacy of ECT in KS skin lesions is limited. In particular, randomized studies and literature reviews are lacking. Therefore, in the present study, a systematic review was performed to assess tumor response and toxicity of ECT. Moreover, the aim of the present study was to evaluate the long-term local control and the impact of ECT on the quality of life (QoL).

\section{Materials and methods}

Research methodology. The protocol of the present systematic review was submitted for registration in the PROSPERO international prospective register of systematic reviews on October 2 , 2019 (9). The analysis was performed based on the Preferred Reporting Items for Systematic Reviews and Meta-Analyses (PRISMA) guidelines (10). The primary endpoint of the analysis was the tumor response to treatment. The secondary endpoints were the adverse events, local control, and impact on QoL.

Bibliographic search. The systematic analysis was carried out using the PubMed, Scopus, and Cochrane databases from the earliest date to October 18, 2019. The search strategies used in the different libraries included were the following: PubMed[((electroporation) OR electrochemotherapy) AND Kaposi sarcoma], Scopus[(TITLE-ABS-KEY (electroporation) OR TITLE-ABS-KEY (electrochemotherapy) AND TITLE-ABS-KEY (Kaposi AND sarcoma))], Cochrane ['electroporation' in Title Abstract Keyword OR 'electrochemotherapy' in Title Abstract Keyword AND 'Kaposi sarcoma' in Title Abstract Keyword]. The reference lists of the selected papers were confirmed to identify additional publications.

Inclusion criteria. The studies with available full text, published in English, reporting on patients with KS skin lesions treated with ECT were included in this systematic review. Both retrospective studies and prospective trials were included. The studies that did not report tumor response or toxicity or those that did not report these outcomes separately from other primary tumors, the case reports, the systematic or narrative reviews, the meta-analyses, the letter-commentaries-editorials, the planning and imaging studies, the surveys, the guideline-recommendations and/or those that reported duplicate data were excluded. In case of inclusion of the same patients in subsequent publications, the most complete or recent article was selected.

Study selection. Following removal of duplicate publications based on their title/abstract, MF and SCa independently reviewed titles, abstracts, and keywords to perform a preliminary selection. In case of differences in the selection, the final decision was obtained through a discussion with a third author (AGM). The full text of all articles potentially suitable for the purposes of this analysis was examined independently by $\mathrm{MF}$ and $\mathrm{SCa}$. In the event of discrepancies, the analysis proceeded as described above.

Data extraction. From the papers selected for inclusion in the review, the data required for the analysis were independently extracted by MF and SCa using a predefined data collection form. The following parameters were included: ECT treatment characteristics (drug, route of administration, number of treatments, number and dimensions of treated lesions), previous and concomitant treatments and their characteristics, tumor response, adverse events, local control and impact on QoL.

The collected data were subsequently confirmed by AGM to identify possible discrepancies among the extracted data. In the event of conflicting data, the final decision was taken by discussion.

Quality assessment. The quality of the papers selected was considered in terms of clear definition of the study population, treatment modality, clear level of the reported outcomes (per lesion or per patient) and missing data.

\section{Results}

Search results. Fig. 1 indicates the PRISMA flowchart of the study selection. The initial search led to the identification of 282 studies (268 following duplicate removal). Five full texts were examined following screening of the titles and abstracts. Following assessment of the latter, three studies were included in the analysis (11-13). One record, which was a letter to the editor, was excluded and the secondary title partially reported on patients was subsequently included in another study from the same centre. All studies were defined as prospective trials by the authors.

Patients and treatment characteristics. The characteristics and results of the analyzed studies are summarized in Table I. Overall, the analyzed papers included 68 patients with KS skin lesions treated with ECT. The number of patients in the studies ranged between 18 and 27 (11-13), while the total number of treated lesions, reported in only two studies was 72 (12) and 532 (11), respectively. Tumor size was not reported in any study.

ECT was performed using intravenous bleomycin in all studies with the number of sessions ranging between one and six. Intravenous bleomycin was administered as intravenous bolus $\left(15,000 \mathrm{IU} / \mathrm{m}^{2}\right)$ based on the ESOPE guidelines (4). Different types of anaesthesia were used as follows: Local, spinal, local-regional and general. The follow-up period was reported as range in all papers. The exact range values were 2.0-50.4 months (11), 6-48 months (12) and 24-68 months (13), respectively. The median follow-up was reported in only one series (18 months) (11). Previous treatments were not reported in one study (13). However, Curatolo et al (11) described previous local or systemic treatments as follows: Systemic chemotherapy $(26.1 \%)$, radiotherapy $(21.7 \%)$, intralesional chemotherapy $(4.3 \%)$ and interferon $(13.0 \%)$. All patients in the series of Latini et al (12) were treated on locally recurrent lesions following cryotherapy.

Two papers reported changes in HHV-8 parameters following ECT. In both series, the viral load and viral blood titre correlated with ECT response. More specifically, the levels of the viral-related parameters declined markedly in patients with complete response (CR) $(12,13)$.

Tumor response and follow-up. In all studies, tumor response was reported on a per patient basis. The CR ranged between 65 and $100 \%$ with an overall response rate (ORR) (CR plus partial response) of $100 \%$ in all studies (11-13). Patients with stable or progressive disease were not reported. The reported 


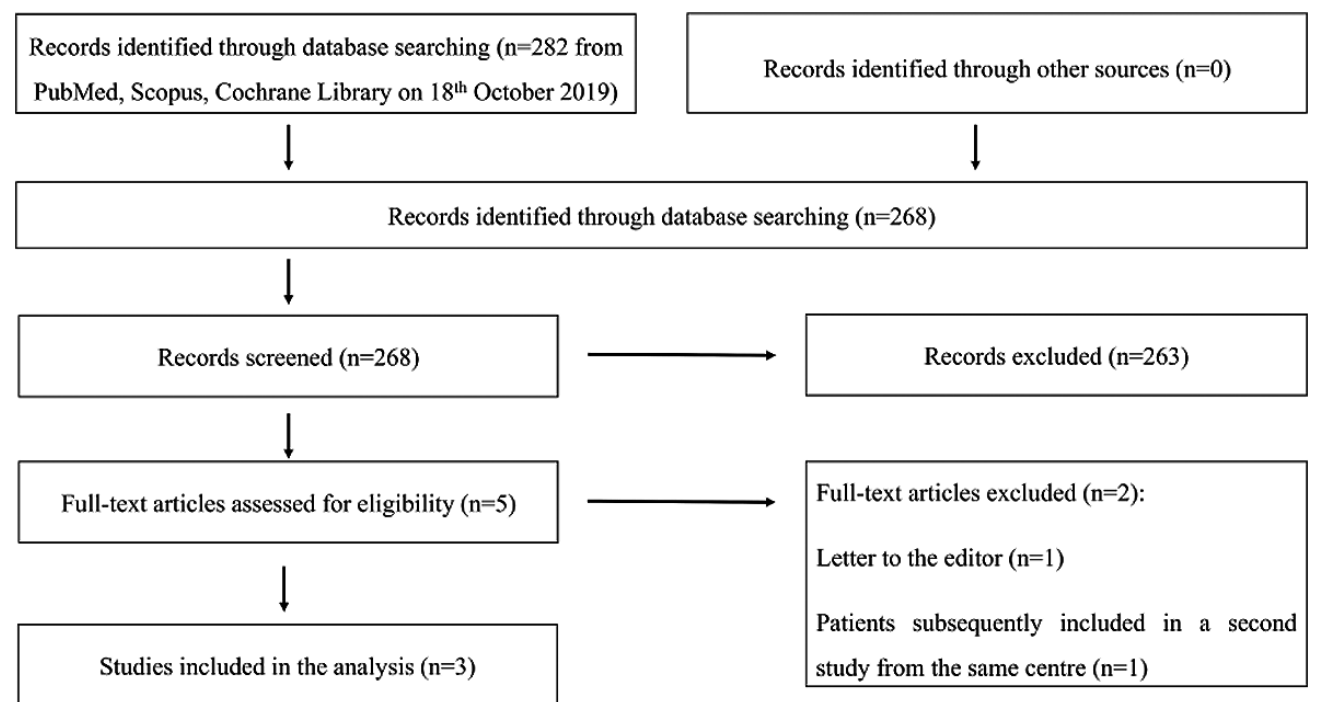

Figure 1. PRISMA flow-chart for the selection of studies.

CR rate after a single ECT session was $60.9 \%$ as reported by Curatolo et al (11) and 74.1\% in the study by Starita et al (13). Moreover, Latini et al (12) reported $88.9 \%$ CR rate after the first ECT session.

All authors evaluated tumor response at four weeks following the last ECT session. However different criteria were used in the present study. In fact, two studies $(11,13)$ used the Response Evaluation Criteria in Solid Tumors and one study used (12) the World Health Organization guidelines.

Toxicity. The incidence of adverse events was low (5.5-8.7\%) in the two studies reporting toxicity $(11,12)$. More specifically, $5.5 \%$ of the subjects exhibited a local ulceration in the study by Latini et al (12), while Curatolo et al (11) reported $8.7 \%$ of subjects with cutaneous infection and $8.7 \%$ with local pain in their series. No cases of systemic toxicity were recorded. One paper (11) used the World Health Organization toxicity scoring system.

Other outcomes. Long term outcome data were analyzed only in one study reporting 2-year local control and overall survival (OS) rates of 76.2 and $74.4 \%$, respectively (11). Moreover, in the same series, a QoL improvement, assessed by means of the Patient Global Assessment (PGA) (14), was recorded in $95 \%$ of patients.

Quality assessment. All studies presented an intermediate risk of bias with regard to patients and treatment description. In fact, only two studies reported the number of treated lesions $(11,12)$, whereas the parameter tumor size was not reported. All studies clearly reported the level of response evaluation and the scoring criteria (11-13). Finally, with regard to the missing data, toxicity was described in two studies $(11,12)$, while only one of them reported the grading criteria (11).

\section{Discussion}

A systematic literature review was performed on ECT in KS skin lesions. The limited number of analyzed studies suggests that this treatment is safe and that it is associated with a very high tumor response rate.

However, the present analysis has one clear limitation. The selection of the studies led to the analysis of only three studies (11-13). Although several series included KS among ECT-treated tumors, in the majority of the cases, the results on $\mathrm{KS}$ alone were not reported separately and therefore they were excluded.

It should be noted that the analyzed studies exhibited a small sample size, a wide variability in terms of lesions/patient ratio and did not report the size of the treated lesions. The latter limitation prevented the assessment of a possible correlation between outcomes and tumor dimension. It is important to note that none of the analyzed papers presented a comparison of the clinical response of the patients with regard to the following parameters: Age, sex, previous treatment history, number of lesions, tumor size and body site. Moreover, although all studies were defined as prospective, none of them described the method of sample size calculation. Furthermore, another limitation is that only one study reported the results in terms of local control and OS (11). Therefore, the investigation of the long-term control of the disease has not been performed thoroughly. Finally, the number of sessions varied widely between the different reports. However, it should be noted that the use of multiple sessions in the same patient was due to several reasons. Firstly, certain patients exhibited a high number of lesions that had to be treated in the same session, whereas other patients required several courses to achieve a satisfactory tumor response after the first one. In addition, patients who developed new lesions between courses were reported.

The results of the analysis suggested a high response rate, which was estimated at $100 \%$ of the ORR rate in all studies. The CR rate, although generally satisfactory, indicated a rather significant variability (65-100\%). These differences may be due to the 'per patient basis' response evaluation combined with the high variability of the lesions/patient ratio noted in the different studies. The response rate 'per patients' was significantly lower in cases of high number of lesions. The probability of response in all lesions was reduced as the number of the latter increased. 
Therefore, the lower CR rate reported by Curatolo et al (11) (65\%) compared with that of Starita et al (13) (100\%)could probably be explained by the higher lesion/patient ratio of the first series. Furthermore, the differences in terms of the response rate may be due to the different evaluation criteria used in the different studies. It is interesting to note that Curatolo et al reported QoL improvement in $95 \%$ of patients, despite the lower $\mathrm{CR}$ rate $(65 \%)$. In addition, in this case it was hypothesized that the large number of lesions per patient reduced the response rate 'per patient' but still produced the reduction in the size in a number of lesions, which was sufficient to improve the QoL (11).

Both studies reported data on toxicity and indicated a relatively low incidence of adverse events $(11,12)$. In particular, the incidence of toxicity was $5.5-8.7 \%$ with regard to the adverse event of each patient. However, based on the incidence of toxicity regarding events per lesion, the results were even more encouraging (overall: $0.8 \%$ ). These results confirmed the safety of ECT in the treatment of skin lesions (5). However, the patients did not undergo combined modality treatments including ECT in the analyzed studies. Therefore, it is impossible to define both tolerability and potential synergistic effects of integrated treatments including ECT in KS skin lesions.

The comparison of ECT with other local therapies in KS demonstrated that radiotherapy was also well-tolerated and associated with favourable results in terms of response (47-99\%) (15-17) and local disease control (18). However, ECT is a repeatable method, as demonstrated by several studies on the treatment of skin neoplasms (7). KS is in the 30th place worldwide with regard to the incidence and in the 29th with regard to mortality. However, there are regions where incidence and mortality of this disease are much higher. For example, 32,446 out of the 41,799 new cases registered in 2018, have been diagnosed in Africa. In addition, 17,659 out of the 19,902 deaths from KS recorded in the same year, were recorded in Africa (19). It is well known that in the African continent the chronic shortage of radiotherapy centres and equipment is associated with the requirement for significant initial investments and with the difficulties in maintaining the equipment. Therefore, the possibility of treating other neoplasms (skin and vulva) with ECT represents a useful integration to the scarce radiotherapy resources available. In this regard, it should be emphasized that the initial costs and maintenance of the ECT are much lower than those of radiotherapy.

Intralesional chemotherapy is also associated with a high response rate $(70.0-98.7 \%)(20,21)$, although this may be lower compared with the results uniformly recorded in our analysis $(100 \%)$.

Another modality of electroporation, is 'irreversible electroporation', a non-thermal tissue ablation technology, which uses short pulses of a high voltage current, without concurrent delivery of chemotherapy, leading to cell membrane disruption and cell death as a result of loss of homeostasis. However, this technique is used only in the treatment of deep tumors and its application on KS has not been reported previously.

The comparison of ECT with systemic chemotherapy is generally used in advanced disease and is based on pegylated liposomal doxorubicin plus paclitaxel or interferon $\alpha-2 a$ or $2 b$. The data demonstrate that the latter is associated with a relatively high response rate (71-100\%) particularly considering this specific advanced disease setting (3). It is important to note that 
these response rates cannot be compared with those of ECT, since the use of systemic chemotherapy is justified only in case of metastatic KS. However, it is speculated that in metastatic diseases, ECT may exert a potential role in oligo-persistent or oligo-progressive skin lesions, notably in symptomatic cases.

In conclusion, the present analysis suggested that ECT may be considered a therapeutic option in KS skin lesions. Further studies are required to improve the existing knowledge on this field. These studies may concern the following topics: i) The comparison between ECT and other local therapies of KS, ii) the role of ECT in skin recurrences following other local therapies, iii) the combination of chemotherapy and palliative ECT in patients with advanced disease and the optimal timing among the two treatments, iv) a long-term evaluation of outcomes, v) the design of clinical trials on KS subjects using ECT in areas with high incidence and mortality from this disease, vi) the development of predictive models of local control in order to favour tailored treatment in patients with different presentations of KS skin lesions.

\section{Acknowledgements}

Not applicable.

\section{Funding}

No funding was received.

\section{Availability of data and materials}

Not applicable.

\section{Authors' contributions}

MF, GM, MB and AGM conceived and designed the study. MF, $\mathrm{SCa}, \mathrm{AZ}$ and AG extracted data from previous studies. MF, AMP, FDT, FD, SCi, LT, PDI and AGM analysed and interpreted the data. All authors wrote the manuscript. All authors read and approved the final version of the manuscript.

\section{Ethics approval and consent to participate}

Not applicable.

\section{Patient consent for publication}

Not applicable.

\section{Competing interests}

FDT is an employee at IGEA. AGM and PDI received grants from IGEA. All other authors declare no competing interests.

\section{References}

1. Ruocco E, Ruocco V, Tornesello ML, Gambardella A, Wolf R and Buonaguro FM: Kaposi's sarcoma: Etiology and pathogenesis, inducing factors, causal associations, and treatments: Facts and controversies. Clin Dermatol 31: 413-422, 2013.

2. Schneider JW and Dittmer DP: Diagnosis and treatment of Kaposi sarcoma. Am J Clin Dermatol 18: 529-539, 2017.
3. Lebbe C, Garbe C, Stratigos AJ, Harwood C, Peris K, Marmol VD, Malvehy J, Zalaudek I, Hoeller C, Dummer R, et al: Diagnosis and treatment of Kaposi's sarcoma: European consensus-based interdisciplinary guideline (EDF/EADO/EORTC). Eur J Cancer 114: 117-127, 2019.

4. Marty M, Sersa G, Garbay JR, Gehl J, Collins CG, Snoj M, Billard V, Geertsen PF, Larkin JO, Miklavcic D, et al: Electrochemothrapy, an easy, highly effective and safe treatment of cutaneous and subcutaneous metastases: Results of ESOPE (European standard operating procedures of electrochemotherapy) study. Eur J Cancer Suppl 4: 3-13, 2006.

5. Cadossi R, Ronchetti $M$ and Cadossi M: Locally enhanced chemotherapy by electroporation: Clinical experiences and perspective of use of electrochemotherapy. Future Oncol 10: 877-890, 2014.

6. Sersa G, Miklavcic D, Cemazar M, Rudolf Z, Pucihar G, Snoj M: Electrochemotherapy in treatment of tumours. Eur J Surg Oncol 34: 232-240, 2008.

7. Esmaeili $\mathrm{N}$ and Friebe M: Electrochemotherapy: A review of current status, alternative IGP approaches, and future perspectives. J Healthc Eng 2019: 2784516, 2019.

8. Cemazar M, Parkins CS, Holder AL, Chaplin DJ, Tozer GM and Sersa G: Electroporation of human microvascular endothelial cells: Evidence for an anti-vascular mechanism of electrochemotherapy. Br J Cancer 84: 565-570, 2001.

9. Centre for Reviews and Dissemination, University of York. PROSPERO: International Prospective Register of Systematic Reviews. https://www.crd.york.ac.uk/prospero. Accessed October 1, 2019.

10. Moher D, Liberati A, Tetzlaff J and Altman DG; PRISMA Group: Preferred reporting items for systematic reviews and meta-analyses: The PRISMA statement. J Clin Epidemiol 62: 1006-1012, 2009.

11. Curatolo P, Quaglino P, Marenco F, Mancini M, Nardò T, Mortera C, Rotunno R, Calvieri S and Bernengo MG: Electrochemotherapy in the treatment of Kaposi sarcoma cutaneous lesions: A two-center prospective phase II trial. Ann Surg Oncol 19: 192-198, 2012.

12. Latini A, Bonadies A, Trento E, Bultrini S, Cota C, Solivetti FM, Ferraro C, Ardigò M, Amorosi B, Palamara G, et al: Effective treatment of Kaposi's sarcoma by electrochemotherapy and intravenous bleomycin administration. Dermatol Ther 25: 214-218, 2012.

13. Starita N, Di Monta G, Cerasuolo A, Marone U, Anniciello AM, Botti G, Buonaguro L, Buonaguro FM and Tornesello ML: Effect of electrochemotherapy on human herpesvirus 8 kinetics in classic Kaposi sarcoma. Infect Agent Cancer 12: 35, 2017.

14. Pincus T, Bergman M, Sokka T, Roth J, Swearingen C and Yazici Y: Visual analog scales in formats other than a 10 centimeter horizontal line to assess pain and other clinical data. J Rheumatol 35: 1550-1558, 2008.

15. Stelzer KJ and Griffin TW: A randomized prospective trial of radiation therapy for AIDS-associated Kaposi's sarcoma. Int J Radiat Oncol Biol Phys 27: 1057-1061, 1993.

16. Caccialanza M, Marca S, Piccinno R and Eulisse G: Radiotherapy of classic and human immunodeficiency virus-related Kaposi's sarcoma: Results in 1482 lesions. J Eur Acad Dermatol Venereol 22: 297-302, 2008.

17. Hamilton CR, Cumming BJ and Harwood AR: Radiotherapy of Kaposi's sarcoma. Int J Radiat Oncol Biol Phys 12: 1931-1935, 1986.

18. Fort M, Guet S, Colson-Durand L, Auzolle C and Belkacemi Y: Role of radiation therapy in non-melanoma cancers, lymphomas and sarcomas of the skin: Systematic review and best practice in 2016. Crit Rev Oncol Hematol 99: 200-213, 2016.

19. GLOBOCAN 2018, Cancer Incidence and Mortality Worldwide: IARC CancerBase. Lyon, France: International Agency for Research on Cancer. http://gco.iarc.fr/today/data/factsheets/popu lations/991-who-africa-region-afro-fact-sheets.pdf. Accessed April 20, 2020

20. Boudreaux AA, Smith LL, Cosby CD, Bason MM, Tappero JW and Berger TG: Intralesional vinblastine for cutaneous Kaposi's sarcoma associated with acquired immunodeficiency syndrome. A clinical trial to evaluate efficacy and discomfort associated with infection. J Am Acad Dermatol 28: 61-65, 1993.

21. Brambilla L, Bellinvia M, Tourlaki A, Scoppio B, Gaiani F and Boneschi V: Intralesional vincristine as first-line therapy for nodular lesions in classic Kaposi sarcoma: A prospective study in 151 patients. Br J Dermatol 162: 854-859, 2010. 